Volume 7, Issue 12, December 2019

DOI: https://doi.org/10.24113/ijellh.v7i12.10218

\title{
Pitch Range in Discourse, for female RHD participants
}

\author{
Dr. Agniva Pal \\ Assistant Professor \\ Amity University \\ India \\ apal@amity.edu
}

\section{Abstract}

This paper conducts a cross sectional study on 4 Bangla speaking RHD (right hemisphere of the brain damaged) participants at the level of discourse (controlled conversation, in lines of narrated stories being retold by the participants), along with 4 age and gender matched controls. We looked at the pitch range in discourse, for these participants. Pitch range is the difference between the highest pitch and lowest pitch points used in the course of a discourse. The ability to modulate the pitch of voice is attributed to the right hemisphere of the brain and damage to it causes inhibitions in the ability to modulate the minimum and maximum pitch. This is important because we convey a lot of information through the use of pitch. Loss of information in speech, in terms of pitch information is very common among stroke patients, if there is damage to the right brain. The loss of pitch information in voice is manifested in a manner such that individuals without training or resources and tools would find it difficult to understand RHD issues.

The study procedure consisted of narrating two short stories to the participants and then requesting them to narrate back the story, which was recorded by the researcher. The 
recordings were analyzed in Praat (a software used in experimental and applied linguistics), where the highest and lowest pitch was found out. Pitch range was found out in Ms. Excel. The 4 participants have been compared to single controls and to the mean of the whole group, both controls and experimental group.

This paper has found out that there is a marked difference between the pitch range of the RHD participants. RHD participants have a pitch range of $163 \mathrm{~Hz}$ in comparison with the control participants who have a pitch range of $192 \mathrm{~Hz}$, proving that RHD participants lose a major chunk of pitch information.

\section{INTRODUCTION}

The right hemisphere of the brain primarily controls the voluntary activities in the left side of the body. Apart from that it imparts personalities to human beings, making us unique and novel and different from each other. The right hemisphere of the brain is also responsible for helping us comprehend the theme of situations, in particular, of discourses. It helps in understanding what is being spoken about, so that we can speak relevant things. The right hemisphere further helps with understanding metaphors and imageries. The right hemisphere is responsible for giving us the sense of perception and when it is damaged the subjects affected can lose the sence of perception. Left side of the body neglect is very common among subjects with right hemisphere damage. The right hemisphere helps use recognise tones in voices as well as other para-linguistic cues in voice which help us understand conversations better. The right hemisphere also gives us the ability to use the frequency modulations, we do, in normal conversations to express various para-linguistic messages along with normal speech (Springer, Deutsch, 1993, Weisenberg, 1935, Rachel and Crow, 2005, Metcalfe, Funnell and Gazzaniga, 1995, Moor, 1982, Robinson, Kubos, Starr, Rao, Price, 1984, Vallar, Perani, 1986, Bihrlea, Brownell, Powelsona and Gardnerc, 1986, Ozonoff, Miller, 1996, Gordon, Hewer, Wade, 1987, Narang, 2009). 
However, in this research paper, we shall take a look at the pitch variation in discourse. We shall compare four right hemisphere damaged female participants (to be henceforth referred to as RHD participants) in comparison with age and gender matched controls. We shall also compare them to the mean of the whole RHD group (mean of all the RHD participants) and the mean of the whole control group.

\subsection{THE PRESENT STUDY}

The present study falls under the broad area of neurolinguistics. It is an empirical study which involves the collection of primary data RHD participants along with age and gender matched normal controls preferably from the same family as the RHD subjects. It is a quantitative study but not a longitudinal study. It is a cross sectional study. The present study would look into pitch variations at the level of discourse.

In the next section we shall do a short literature review of relevant literature to understand the nature and intensity of damage. Throughout my field study, I have come across various RHD participants and it was a clear fact that the lack of the ability to use pitch variations in speech, makes it difficult for the listener to understand speech, as well as it is done with normal people. The literature review shall discuss similar issues, faced by researchers before. We shall discuss the research methodology in the section following literature review and it would elaborate the various steps taken throughout the field study, for studying the participants, as well as controls from the same family or similar socio-economic backgrounds. This would be followed by a section called case study, which would discuss the background of the RHD participants included in this study. The final section would house the discussions and analysis found from the data presented in the subsequent sections. 


\section{LITERATURE REVIEW}

The paper Characterizing sentence intonation in a right hemisphere-damaged population by Behrens SJ, characterized the sentence intonation of RHD patients. The study focused on a greater number of acoustic parameters and the recordings were elicited under natural circumstances. Eight RHD speakers and seven normal control participants produced declarative and imperative sentences as well as yes-no and wh-questions. The slope of F0 change, linearity of pitch contour, and variance of F0 points were calculated for each utterance. The same was calculated for the preterminal and the terminal contour separately. The contours in RHD were less linear and flatter in F0 decline than normal controls for the declarative sentences. The response to yes or no questions from brain damaged patients differed a lot from the response from normal controls. Patients displayed a smaller F0 dispersion around a mean F0. 'Preterminal range values were more restricted for patients' utterances of yes-no questions, while terminal properties between groups differed for three of the four sentence types examined.' Hence it can be discerned that patients with damage to the right brain have some problem in modulating their fundamental frequency. (Behrens, 1989, 181-200)

The pitch in sentences are measured by looking into the pitch range of vowels. This requires the f0 for vowels to be found out at each levels of vowels. It has been seen that RHD participants have lesser variation in f0 range than normal controls. In the article Right Hemisphere Language Comprehension: Perspectives From Cognitive Neuroscience by Mark Jung Beeman, C. C. (1997).. Mahwah, New Jersey: Psychology Press, it is mentioned that the Right Hemisphere is not directly responsible for language but without the well-functioning of the Right Hemisphere it is impossible to speak the way we do. The Right Hemisphere is responsible for helping speakers with the f0 of speech as well as the ability to hold on to conversations and their pragmatic essences. (Mark Jung Beeman) 
According to the book, Language and the Brain by Loraine K Obler and K Gjerlow, participants with RHD have lower f0 variation in their speech in normal discourse. RHD participants generally speak without much variations in their speech. A nomal control on the other hand has good variation in speech. A person listening to an RHD subject would have problems in understanding the mood and emotions of a person through the intonations in the sentences. Generally we know if a person is sad, angry, happy, disgusted by the intonation and pitch variations in a sentence but the same in an RHD subject is difficult to discern because of the damage caused to the right hemisphere of the brain. (Loraine K Obler, 1998, 20)

According to the article by Penelope S Myers, Analysis of Right Hemisphere Communication Deficits: Implications for speech pathology, damages to the parietal region of the right hemisphere makes a subject lose tonal range in speech. Sentences of such individuals either verbal or non-verbal do not contain the emotion originally supposed to be there. The author also suggests that the 'witticisms' of such participants are also conveyed in a 'flat tone'. (Myers, Analysis of Right Hemisphere Communication Deficits: Implications for speech pathology, 1998, 1)

According to the book Right hemisphere damage: Disorders of communication and cognition by Penelope S Myers (Myers), participants with RHD have problems in understanding the intonation in sentences they hear. They also face problems in using proper intonations in their sentences. Participants have difficulty in understanding the pitch in speech they listen to and judge the sentences based only on the content of the sentences. Generally, we judge a sentence and comprehend it according to Grice's Maxims (Grice) but we also comprehend a sentence according to the intonation patterns used.

As seen in a large number of studies, the pitch levels in the sentences of participants affected with RHD have been seen to be lesser. Participants speak within a limited range and do not cover the full range of frequency normal controls can. It has been noticed that RHD 
participants have a lesser variation for pitch in sentence intonation for declarative, interrogative and imperative sentences. Every human being has a fundamental frequency of their own but their pitch in actual speech depends on a lot of factors. Pitch patterns in a sentence can be drastically different depending on if the sentence being spoken is a statement or a question or an order. We shall compare the pitch variations in the intonations for declarative statements in RHD participants in comparison with normal controls. Various other factors like mood of the speaker, the topic of discourse, the physical environment the speaker is in, the physical disorders of a person including brain damage and many other factors can change the pitch range in speech for a human being. (Behrens, 1989, Eriksson, 1994, Myers, 1998, Obler, 1998, Ahlsen, 2006)

Praat is a software which can be used (Praat: Home) to find out formants, pitch, intensity and other acoustic data from sound files while working with phonological data. Praat can also be used to segment sounds, de-noise sound files and to monitor sound spectrograms for further analysis. Other tools like palatography and electropalatography can help understand the active and passive articulators and the exact regions of articulation.

Agniva Pal (2016) unpublished M.Phil thesis on Language and Speech of the RHD, presents the study 18 right hemisphere damaged participants in comparison with their age and gender matched normal controls. It studies the deviations at the level of discourse, frequency at the level of sentences and vowels as used in words at the level of speech. At the level of discourse it studied the duration of discourse, the fluency in discourse, comprehension and digressions in speech. It included some personal and subjective views from the researcher regarding the facial expressions and emotional quotients. At the level of sentences, it studied the fundamental frequency as a measure for pitch levels in declarative sentences for continuous speech. It studied the pitch range through the study of maximum and minimum frequency in continuous speech. At the level of vowels as in words. The acoustic space of vowels in cases 
of RHD and Controls was studied in detail using all peripheral vowels in the Bengali language. It is interesting to note that in some cases the acoustic space of vowels was reduced to as small as $9 \%, 13 \%, 23 \%, 43 \%$ to $70 \%$ and $90 \%$. In the cases with more than $70 \%$ acoustic space, the deviations were not audible without tools and instruments. It is also important to notice that no major difference was found between the RHD group and the control group in terms of vowel duration.

The Glasgow Coma scale is a test, which can be administered only by clinical persons on patients and participants to understand the extent of coma. GCS can actually help a researcher understand how terminally ill a participant is. The scores are distributed as such: Eye (4), Verbal (5), and Motor (6). The maximum score is 15 . A score of under 11 is considered critical. A score under 8 is considered fatal. A score under 15 needs urgent attention and care. (Glasgow Coma Scale/Score (GCS)) The NIMHANS neurophysiological battery serves a similar purpose. (Rao SL, Subhakrishnan DK, Gopulkumar K, 2004)

The Mini Mental State Examination or MMSE is a very common way to test problems of memory loss and other related mental abilities. It lets a researcher understand if an individual is suffering from dementia or not. Prior medical records of a person can further substantiate the results of an MMSE exam. There are various questions in an MMSE exam, the full marks being 30. A score of 27 or more is expected from undamaged individuals with no dementia. There is a hindi version of the MMSE exam available in India called the Hindi Mental State Examination (Ganguli et all, 1993). (The MMSE test)

\section{RESEARCH METHODOLOGY}

The present study is a neurolinguistic study, which uses tools and methodologies from general linguistics as well as neurolinguistics. The present research also uses audio recordings to find out the pitch variations in Bangla speaking cases of Right Hemisphere 
Damage through the use of Praat, to analyze the formants, the pitch and other acoustic data in the recordings. At the level of discourse, the participants were required to listen to two short stories and then narrate the same story back to the researcher, being as close to the original story as possible.

Linguistic fieldwork requires the researcher to make the participants feel comfortable. It is absolutely a necessity that the participants feel normal and stable for the researcher to record unbiased opinions, recordings or videos. If the participant is not speaking the way he or she speaks normally, it would entail some prejudice in the data for that research. It can skew the entire data of the research. (Max Planck) Under circumstances when the participant being researched on, is a tribal or from an extremely backward region, they must be made to feel comfortable. A working rapport with the participant and the researcher can ease up the process of data collection largely.

\subsection{PARTICIPANT SELECTION}

Preferably, monolingual Bangla speaking participants who have damage to the right hemisphere of the brain, have been selected. Controls chosen are generally members of the same family with age and gender matching. In case no one from the same family was available for the research according to the parameters mentioned above, age and gender matched control participants were chosen from the same socio- economic background.

\subsection{INCLUSION AND EXCLUSION CRITERIA}

Primarily, the research data elicitation began with random sampling. The inclusion criteria had been set after a few random samplings. The Mini Mental State Exam ensured the current state of the participants, both RHD and healthy participants, and a score of 25-30 would ensure, that they are not suffering from any other sort of disorders which might sabotage the 
data. The Glasgow Coma Scale with score of at least 11 would also ensure similar levels of severity of all participants and that no participant is under coma, which might sabotage the data. The exclusion criteria would make sure that participants in the ICU would not be selected, or participants with extreme disabilities. The other criteria have been determined after the random sampling phase.

\subsection{ETHICAL CLEARANCE}

Any research involving human participants, being conducted by JNU students require an ethical clearance from the JNU Institutional Ethics Review Board (IERB). A detailed description of the research proceedings and the involvement details of the participants have to be stated to the JNU IERB while presenting one's research proposal to be granted an ethical clearance. The ethical clearance for the present research, granted by IERB, JNU has been attached in the appendix. For working with human participants, other than being granted an ethical clearance from JNU IERB, a researcher also needs a PIS ICF form. When a participant agrees to provide information for a certain research, he or she has to sign a form with detailed information about the research (including information like a brief introduction, research objectives, nature of involvement of the subjects and how the information will be used).

\subsection{FIELD STUDY}

To acquire a steady flow of participants, Bangur Institute of Neurosciences, Kolkata had been approached. Dr, Goutam Ganguly, from the above-mentioned institute was chosen to help with the present research. The participant selection procedure according to the inclusion and exclusion criteria have all taken place under his supervision. Participants have been documented in details. A specific format for documenting participants was created and used. Such forms for all participants have been filled up with details of the subject like the place of 
lesion or lesions, the time post incidence, the present condition, the symptoms and so on. In the primary information section, name, age and gender of the subjects have also be documented, although this is purely for documenting the data and related purposes. The names of the participants have not been used in the present research; instead codes have been used.

\subsection{CODING PARTICIPANTS}

Participants have been coded according to the following table:

\begin{tabular}{|l|l|l|l|l|}
\hline Serial number & Name initials & Age & Gender & R / C \\
\hline 00 & AB & 50 & M & R \\
\hline 01 & AC & 55 & F & C \\
\hline
\end{tabular}

The above table explains the process of coding the names for the participants in the present study. The unique serial number for every participant is an 8-digit code. The first two digits are allotted according to alphabetical order of the participants. The initials of the name have engaged the next two digits. The next two digits have been used by the age followed by a single digit for gender. The last digit is either R (signifying RHD) or C (signifying controls). For example, 00AB50MR, 01AC55FC as stated in the table above.

\subsection{TOOLS AND INSTRUMENTS}

This section houses all the various tools, either technical or linguistic. Linguistic research requires recording equipment and softwares. Linguistic research requires the ability to transcribe spoken speech for further analysis using various softwares like Praat, Goldwave and Elan. 
Praat for Mac and Windows (Boersma) has been used for analyzing the formants and pitches of the recordings. Praat is a free software for analyzing and doing phonetics on personal computers. (Praat: Home)

Microsoft Excel was primarily used to save the primary data after being processed from Praat. Microsoft Excel, a program based on spreadsheets helps in creating the charts necessary to compare two groups of data. (Excel: Create Order)

The main research has been written with the help of Microsoft Word. Microsoft Word is an efficient document handling program. (Word: Write On)

Primary recording has been conducted with a ZOOM H1 field recorder. It is a handy microphone with functions like low cut and PCM lossless recording formats.

The pitch variations in the discourse have been found by following th following set of processes:

1. Record the stories from the participants

2. Open the files in Praat

3. Find out the maximum pitch used in the course of the discourse recording

4. Find out the minimum pitch used in the course of the discourse recording

5. Find out the range by subtracting the minimum from the maximum pitch

6. Create graph with the info

\subsection{THE STORIES}

The following stories were narrated to the participants in Bangla.

\section{The Hart and the Hunter}

The Hart was once drinking from a pool and admiring the noble figure he made there.

"Ah," said he, "where can you see such noble horns as these, with such antlers! I wish I had legs more worthy to bear such a noble crown; it is a pity they are so slim and slight." 
At that moment a Hunter approached and sent an arrow whistling after him.

Away bounded the Hart, and soon, by the aid of his nimble legs, was nearly out of sight of the Hunter; but not noticing where he was going, he passed under some trees with branches growing low down in which his antlers were caught, so that the Hunter had time to come up.

"Alas! Alas!" cried the Hart: "We often despise what is most useful to us"

\section{The Frogs Desiring a King}

A few frogs lived happily in a marshy swamp that was perfect but then they started to look for a king who would rule them. They prayed to God to give them a king. God sent them a big block of wood and it fell right into the marsh. At first, they were afraid of its size but then they slowly got used to it and realized it was not moving. They finally, climbed to the top of it and conquered their king. They prayed to God again, this time asking for a live king who would actually rule over them. Now this made Jove angry, so he sent among them a big Stork that soon set to work gobbling them all up.

Then the Frogs repented when too late.

\section{CASE STUDY}

This section displays important information about every RHD participant. It primarily provides primary information about the female RHD participants. This section primarily begins with the codified name, followed by the control participant the RHD participant is being compared with. It also includes the MMSE and GCS scores, the time post incidence and a short description of the condition of the participant. The area of damage in the brain follows the above-mentioned sections. 


\subsection{CASE STUDY 1}

\section{$\underline{\text { PRIMARY INFO }}$}

Codified Name: 22CS62FR

Female, 62 years of age, at the time of recording and case study.

Age and gender matched control: 03NS64FC

Time since incidence: 3 months back, at the time of recording.

\section{PARTICIPANT INFORMATION}

Condition of participant: The participant was fully conscious, having scored more than 11 on the GCS. She also scored 27 on the MMSE.

The speech of the participant was slightly slurred but was understandable. The total loudness of her speech went down, according to her family members.

The doctor also performed a test in which he held both her hands in his hands and then asked her to keep them where he is holding them, after he lets her hands go. She could maintain the position, but her left hand fell a fair bit, before she regained control. According to the doctor, these are subtle signs of the stroke to the right hemisphere.

Observations made by doctor: The doctor mentions that she has no agraphia, no dementia, as well as no alexia. Participant is not hypertensive.

Region of damage: A small sub-acute ischemic infarct at right capsule-thalamic region.

\subsection{CASE STUDY 2}

\section{$\underline{\text { PRIMARY INFO }}$}

\section{Codified Name: 23GD65FR}

Female, 65 years of age, at the time of recording and case study.

Age and gender matched control: 03NS64FC 
Time since incidence: approximately 6 months back, at the time of recording.

\section{$\underline{\text { PARTICIPANT INFORMATION }}$}

Condition of participant: The participant was fully conscious, having scored more than 11 on the GCS. She also scored 28 on the MMSE.

The speech of the participant was not slurred and was completely understandable. The total loudness of her speech went down, according to her family members and could be noticed while making conversations with her, as well as the recording sessions.

The family of the participant assured that she was facing no problems in eating. The doctor also performed a test in which he held both her hands in his hands and then asked her to keep them where he is holding them, after he lets her hands go. She could maintain the position, but her left hand fell a fair bit, before she regained control. According to the doctor, these are subtle signs of the stroke to the right hemisphere.

Observations made by doctor: The doctor mentions that she has no agraphia, no dementia, as well as no alexia. Participant is hypertensive.

Region of damage: An acute lacunar infarct at the right parietal lobe.

\subsection{CASE STUDY 3}

\section{PRIMARY INFO}

Codified Name: 24NB50FR

Female, 50 years of age, at the time of recording and case study.

Age and gender matched control: 06RB56FC

Time since incidence: approximately 4 months back, at the time of recording. 


\section{$\underline{\text { PARTICIPANT INFORMATION }}$}

Condition of participant: The participant was fully conscious, having scored more than 11 on the GCS. She also scored 26 on the MMSE.

The speech of the participant was slightly slurred, due to left hemiparesis, but was understandable.

On being asked to name colours, she could name them correctly.

The doctor also performed a test in which he held both her hands in his hands and then asked her to keep them where he is holding them, after he lets her hands go. She could maintain the position of the right hand, but her left hand fell down. She kept trying, but she could barely move her left hand.

Observations made by doctor: The doctor mentions that she has no agraphia, no dementia, as well as no alexia. Participant is hypertensive.

Region of damage: Haemorrhage in the right basal ganglia.

\subsection{CASE STUDY 4}

\section{$\underline{\text { PRIMARY INFO }}$}

Codified Name: 25UG58FR.

Female, 58 years of age, at the time of recording and case study.

Age and gender matched control: 07SB60FC

Time since incidence: approximately 1 year back, at the time of recording.

\section{$\underline{\text { PARTICIPANT INFORMATION }}$}

Condition of participant: The participant was fully conscious, having scored a perfect 15 on the GCS. She also scored 29 on the MMSE. 
The speech of the participant was not slurred, and was completely understandable.

She mentioned that she has no problems in eating.

The doctor also performed a test in which he held both her hands in his hands and then asked her to keep them where he is holding them, after he lets her hands go. She could maintain the position of both the hands.

Observations made by doctor: The doctor mentions that she has no agraphia, no dementia, as well as no alexia. Participant is not hypertensive.

Region of damage: Infarct at the right basal ganglia.

\section{ANALYSIS AND DISCUSSIONS}

In this section, we will take a look at the pitch variations in the discourse recordings in a graphical format. The following graph displays the pitch variations in the discourse recordings. The vertical axis marks the frequency of the discourse recordings, in this case the pitch range. The horizontal axis has 8 participants. This paper compares four female RHD participants with four age and gender matched controls. The last two columns are the means of the RHD group and control group, respectively. 


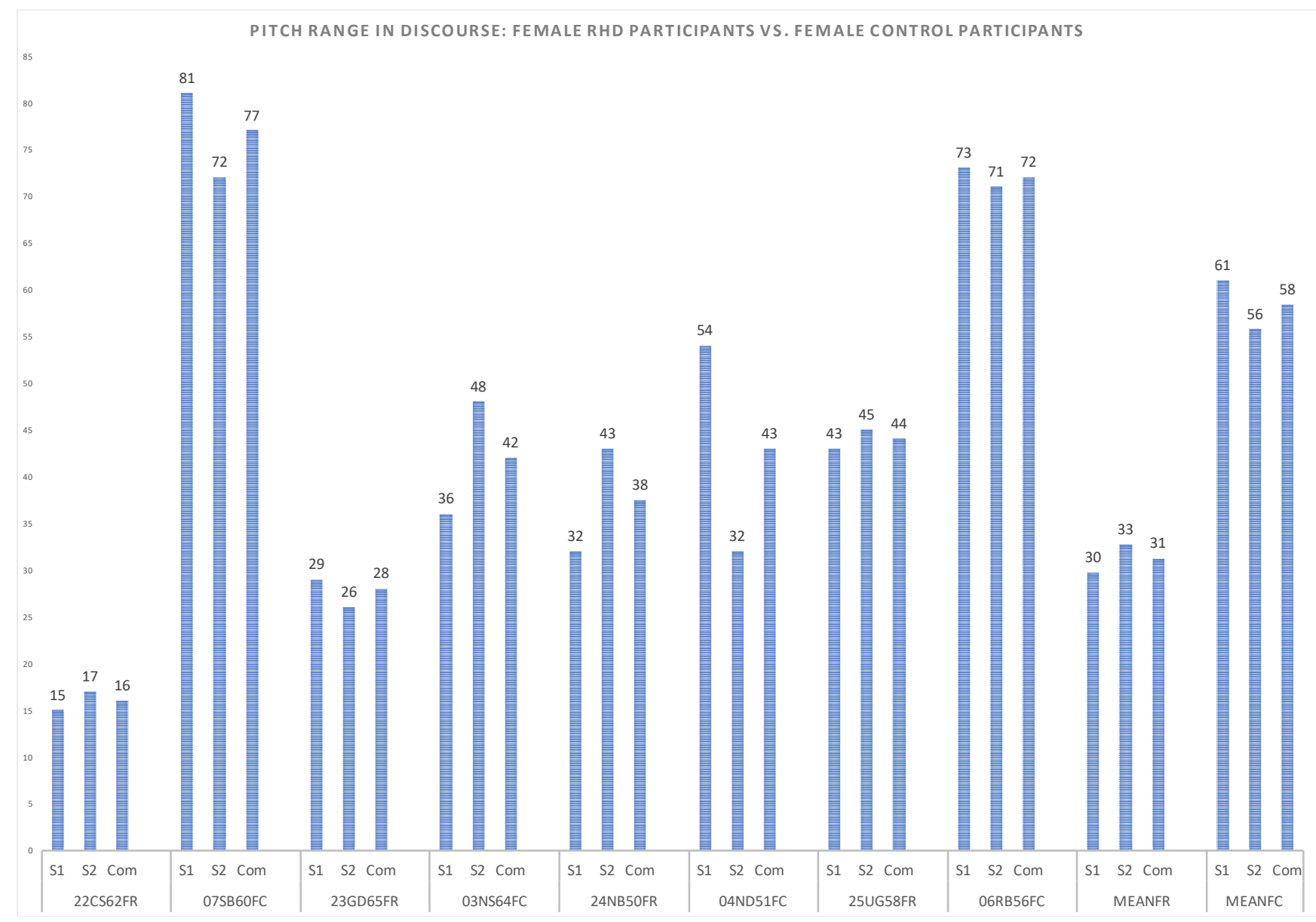

We can discern the following from the figure above:

1. 22CS62FR has a pitch range of $15 \mathrm{~Hz}$ in the first story in comparison with 07SB60FC, who has a pitch range of $81 \mathrm{~Hz}$. The difference between the individual RHD and control participant is significant and displays a noticeably diminished pitch range.

2. 22CS62FR has a pitch range of $17 \mathrm{~Hz}$ in the second story in comparison with 07SB60FC, who has a pitch range of $72 \mathrm{~Hz}$. The difference between the individual RHD and control participant is significant and we can notice a much smaller pitch range in the RHD participant. 
3. 22CS62FR has a pitch range of $16 \mathrm{~Hz}$ in the mean of the stories in comparison with 07SB60FC, who has a pitch range of $77 \mathrm{~Hz}$. The difference between the individual RHD and control participant is highly significant.

4. 23GD65FR has a pitch range of $29 \mathrm{~Hz}$ in the first story in comparison with 03NS64FC, who has a pitch range of $36 \mathrm{~Hz}$. The difference between the individual RHD and control participant is insignificant.

5. 23GD65FR has a pitch range of $26 \mathrm{~Hz}$ in the second story in comparison with 03NS64FC, who has a pitch range of $48 \mathrm{~Hz}$. The difference is significant.

6. 23GD65FR has a pitch range of $28 \mathrm{~Hz}$ in the mean of the stories in comparison with 03NS64FC, who has a pitch range of $42 \mathrm{~Hz}$. The difference between the individual RHD and control participant is not highly significant but as we have seen in the second story, the difference was substantial.

7. 24NB50FR has a pitch range of $32 \mathrm{~Hz}$ in the first story in comparison with 04ND51FC, who has a pitch range of $54 \mathrm{~Hz}$. The difference between the individual RHD and control participant is significant and highlights the decreased pitch range in the RHD participant.

8. 24NB50FR has a pitch range of $43 \mathrm{~Hz}$ in the second story in comparison with 04ND51FC, who has a pitch range of $32 \mathrm{~Hz}$.

9. 24NB50FR has a pitch range of $38 \mathrm{~Hz}$ in the mean of the stories in comparison with 04ND51FC, who has a pitch range of $43 \mathrm{~Hz}$. Here, we notice that the difference between the two pitch ranges are not significant.

10. 25UG58FR has a pitch range of $43 \mathrm{~Hz}$ in the first story in comparison with 06RB56FC, who has a pitch range of $73 \mathrm{~Hz}$. The difference between the individual RHD and control participant is significant but we must also notice that the RHD participant has a pitch range much higher than the other RHD participants and in this case the control participant has a pitch range much higher than the mean. 
11. 25UG58FR has a pitch range of $45 \mathrm{~Hz}$ in the second story in comparison with 06RB56FC, who has a pitch range of $71 \mathrm{~Hz}$. The difference between the individual RHD and control participant, again is significant because the RHD participant has a pitch range much higher than the other RHD participants and in this case the control participant has a pitch range much higher than the mean.

12. 25UG58FR has a pitch range of $44 \mathrm{~Hz}$ in the mean of the stories in comparison with 06RB56FC, who has a pitch range of $72 \mathrm{~Hz}$. The difference between the individual RHD and control participant is significant proving that the RHD participant has much lesser pitch variation in speech but we must also notice that the RHD participant has a pitch range much higher than the other RHD participants and in this case the control participant has a pitch range much higher than the mean.

13. 59MEANFR has a pitch range of $30 \mathrm{~Hz}$ in the first story while 57MEANFC has a pitch range of $52 \mathrm{~Hz}$ in the first story. We can see a significant difference in the pitch range.

14. 59MEANFR has a pitch range of $33 \mathrm{~Hz}$ in the second story while 57MEANFC has a pitch range of $50 \mathrm{~Hz}$ in the second story. Again, we can see a significant difference in the pitch range.

15. 59MEANFR has a pitch range of $31 \mathrm{~Hz}$ in the mean of the two stories while 57MEANFC has a pitch range of $51 \mathrm{~Hz}$ in the mean of the two stories. This means that the RHD participant has much less pitch range in speech, while talking normally, bringing down their ability to convey pitch information through speech.

\section{Conclusion}

We can discern a huge difference in pitch variation between the RHD mean and the control group mean, which, in a way is a proof to the literature review done previously, stating that people affected with damage to the right hemisphere of the brain have a chance of losing pitch variations in their speech, making it more difficult for them to express emptions and 
expressions, in comparison with age and gender matched controls. It also makes it much more difficult for the listener to understand speech from an RHD participant because, the RHD participant provides less intonation information with their speech, in comparison with normal people. Here, it should be noted that exceptional cases, where the control participant has a range, lesser than the RHD participant, might stem from reasons like:

1. The participant being bored in general

2. The participant being tired in general

3. The natural inclination or habit of speaking in a disinterested way (because that is when we speak with less expressions, hence less pitch)

4. The fact that the participant might be tired

5. The fact that the participant, being from the same family as the RHD participant, might have an undiagnosed brain damage.

We can also discern the following from this research paper:

1. RHD participants have a minimum range of $16 \mathrm{~Hz}$ in case of $22 \mathrm{CS} 62 \mathrm{FR}$ and $44 \mathrm{~Hz}$ in case of 25UG58FR.

2. After referring to the case studies we might also conclude that 22CS62FR, who has been recorded 3 months back, has much more damage and has $16 \mathrm{~Hz}$ pitch range while 25UG58FR, who has been recorded after 1 year from the incidence, has much lesser damage and has a pitch range of $44 \mathrm{~Hz}$.

3. We can see a mean pitch range of $31 \mathrm{~Hz}$ in the RHD participants whereas the control group participants have a mean of $51 \mathrm{~Hz}$. We can see a pitch range difference of $20 \mathrm{~Hz}$, which is a lot, considering that $22 \mathrm{CS} 62 \mathrm{FR}$ has a pitch range of $16 \mathrm{~Hz}$. 
Bibliography

Mark Jung Beeman, Christine Chiarello. Right Hemisphere Language Comprehension:

Perspectives From Cognitive Neuroscience. Mahwah, New Jersey: Psychology Press, 1997.

Myers, Penelope S. Right hemisphere damage - Disorders of communication and cognition. London: Singular Publishing Group, 1999.

Grice, H.P. "Logic and Conversation," Syntax and Semantics. Academic Press, 1975.

Praat: Home. 4 March 2015. <http://www.fon.hum.uva.nl/praat/>.

Max Planck. 2017 Feb 11. <https://www.eva.mpg.de/lingua/tools-at-lingboard/tools.php>.

The MMSE test. 11 Feb 2017.

<https://www.alzheimers.org.uk/info/20071/diagnosis/97/the_mmse_test>.

Glasgow Coma Scale/Score (GCS). 11 Feb 2017. <https://www.mdcalc.com/glasgow-comascale-score-gcs>.

Boersma, Paul, and David Weenink. "Praat, a system for doing phonetics by computer." (2001): 341-345.

Goldwave Home. 26 May 2015. <www.goldwave.com/>.

Excel: Create Order. 4 March 2015. <https://products.office.com/en-us/excel>.

Word: Write On. 4 March 2015. <https://products.office.com/en-us/word>. 